\title{
Virtual Seismometers in the Subsurface of the Earth from Seismic Interferometry
}

Andrew Curtis ${ }^{1,2}$, Heather Nicolson ${ }^{1,2,3}$, David Halliday ${ }^{1,2}$, Jeannot Trampert $^{4}$, Brian Baptie ${ }^{2,3}$

${ }^{1}$ School of GeoSciences, University of Edinburgh, Grant Institute, West Mains Rd., Edinburgh EH9 3JW, UK.

${ }^{2}$ ECOSSE (Edinburgh Collaborative of Subsurface Science and Engineering), UK

${ }^{3}$ British Geological Survey, Murchison House, Kings Buildings, West Mains Road, Edinburgh, UK

${ }^{4}$ Faculteit Aardwetenschappen, University of Utrecht, Postbus 80.021, Utrecht, NL.

Correspondence to Andrew.Curtis@ed.ac.uk 
The Earth's interior can be imaged by analysing the records of propagating seismic waves. However, the global array of permanent seismometers that record seismic energy is confined almost exclusively to land-based sites. This limits the resolution of subsurface images, and results in relatively few local measurements from areas of great geological and tectonic interest (for example, the mid-ocean ridges and the Tibetan plateau) ${ }^{1}$. Here we use an unconventional form of seismic interferometry ${ }^{2-5}$ to turn earthquakes into virtual seismometers located beneath the Earth's surface. Seismic waves generated by one earthquake lead to transient strain in the subsurface at other locations around the globe. This strain can be quantified from seismograms of independent earthquakes that have occurred in those locations. This technique can therefore provide information on the subsurface strain in regions of the globe that lack instrumental networks. Applying our method to earthquakes in Alaska and the southwestern United States, we show that the information that can be obtained from these earthquakes about other such events is consistent with that provided by instrumental seismometers. Our approach may allow realtime, non-invasive, subsurface seismic strain monitoring, particularly in areas remote from instrumental networks.

To interrogate the Earth's subsurface at depths greater than a few kilometres, traditional seismology analyses seismic wave energy from earthquakes. Other energy recorded in seismograms, such as ambient Earth oscillation, is considered noise and is excluded from analysis. Since 2003 , however, methods of seismic interferometry have been developed to synthesize impulsive source seismograms from ambient noise recorded at two seismic receivers2. These seismograms simulate the situation where energy from a relatively impulsive, imagined or 'virtual' source occurring at the location of one receiver was recorded by the other.

In Fig. 1a we show a sketch of source-receiver geometries used for interferometry. Recordings of each boundary source at the pair of receivers are cross-correlated, then integrated (summed) over all sources. The result gives the seismogram that would have been recorded at one receiver if the other receiver had instead been a source. Theoretically such seismograms can be constructed provided the sources of seismic energy are distributed to form an enclosing boundary of sources, although the latter geometrical constraint can often be relaxed in practice (see below). Given a suitable receiver geometry, interferometry obviates the need for actual earthquake sources for 
imaging the Earth ${ }^{2,6-10}$. Variations of interferometric theory work in attenuative media, for diffusive, electromagnetic and electro-kinetic energy propagation ${ }^{11-14}$, when using active rather than passive sources of elastic or electromagnetic energy ${ }^{15-21}$ and can be used to create novel methods for wavefield simulation ${ }^{22-24}$.

Although in principle interferometry frees seismologists from constraints imposed by the global distribution of earthquakes, which is strongly biased towards active margins and mid-ocean ridges, the global receiver distribution is also strongly biased (Supplementary Fig. S2). More than two-thirds of the Earth's surface is covered by liquid water or ice, rendering receiver installation difficult and expensive. Even many land-based areas have few receivers because they are geographically or politically inhospitable (for example, the Tibetan and Andean plateaus, Central Africa). Hence, most of the Earth's subsurface can only be interrogated using long earthquake-receiver or receiver-receiver paths of energy propagation. This provides relatively poor spatial resolution of some of the most intriguing tectonic, geological and geophysical phenomena such as mid-ocean ridges and plate convergence zones, and consequently there is a need for data to be recorded locally to such phenomena.

By taking the reciprocal of its usual form, in the Supplementary Methods we show that the impulsive source form of interferometry can also be used in the opposite sense: to turn any energy source into a virtual sensor. In this form, we apply interferometry using sources enclosed within a boundary of receivers (Fig. 1b). This approach is related to that of ref. 25 , but they used the passive noise (rather than impulsive source) form of interferometry. We demonstrate a substantial improvement over their results. It has been shown that it is not always necessary to have an entire enclosing boundary, provided sources are located within a cone around the extension of the inter-event path $^{26}$ (Fig. 1b). We make use of the latter geometrical approximation in our examples.

To illustrate this new method simply, we use real station recordings of the 2008 Sichuan earthquake from the Caltech Regional Seismic Network to construct seismograms recorded by two virtual receivers constructed from other earthquakes, one in the Alaskan subduction zone and one in California, respectively. These virtual receivers and real stations lie approximately on a great circle with the Sichuan earthquake (Fig. 2e). It is assumed that seismic energy will travel along this path between the various chosen locations. For each Californian station located around the great circle path (the configuration of Fig. 1c) the seismograms for the Sichuan and 
virtual receiver earthquakes are cross-correlated, then the resulting cross-correlations are summed. In Fig. 2 we show the real recordings of the Sichuan earthquake at stations located close to each virtual receiver (Fig. 2a and c) and the resulting virtual receiver records (Fig. $2 b$ and d).

The real and virtual traces should not be exactly the same because the virtual receiver records strain whereas real receivers measure displacement (Supplementary Methods). In addition, the stations used for comparison are not collocated with the virtual receivers. Nevertheless, the similarity between the real and virtual receiver recordings is clear.

As the match in Fig. 2 is not perfect, we consider test cases using earthquake and receiver geometries that allow a deeper analysis of the method. The Supplementary Methods shows that virtual sensors inherit the spatio-temporal response function of the original earthquake source: those constructed from purely normal and purely thrust earthquakes thus measure strains in a vertical-horizontal plane, whereas those from strike-slip earthquakes measure strain in the purely horizontal plane. Those constructed from subsurface explosions or implosions measure volumetric expansion of the rock mass (the solid-body equivalent of a pressure sensor in a fluid) ${ }^{27}$. Supplementary Table S1 summarizes the strain components measured for each canonical earthquake or explosive source mechanism.

Figure 3 shows earthquakes and stations used for verification. Two earthquakes with approximately canonical (strike-slip and normal) moment tensor sources were chosen to be converted into virtual sensors because (1) seismometers (MLAC and R06C) exist in their local vicinity for comparison, (2) they had a well-constrained moment tensor source mechanism, (3) they had the lowest possible magnitude subject to constraints (1) and (2) and hence are spatially and temporally as localized as possible, reducing associated relative phase differences between recordings on seismometers and virtual sensors (the source times used for the seismometer recordings are those from the International Seismology Centre (ISC) catalogue; no centroid moment tensor (CMT) source mechanisms and timings were available).

We analysed seismograms from two other earthquakes recorded on these virtual sensors, one chosen to have the source-virtual sensor path aligned roughly east-west, the other chosen to have a roughly perpendicular path. We compare strain recordings of these events on the virtual sensors with estimates of strain constructed from 
recordings of particle velocity from the neighbouring seismometers (see the Methods section below).

Virtual sensors were constructed by integrating (summing) unweighted recordings from a subset of other available seismometers that did not include either comparison seismometer (Supplementary Methods, Equation (S18)). Each subset consisted of seismometers within a cone around the propagation path direction at the virtual sensor (Fig. 3), as these are expected to record the main energy that integrates constructively within the virtual receiver seismogram ${ }^{28}$. Conclusions herein are robust to changes in the subtending angle of the cone.

Figure 4 shows earthquake 1 recorded by the virtual sensor constructed from the N-S oriented normal fault. This virtual receiver measures the difference between the e33 and e11 components of the strain. Although we do not have a comparison measurement for the $e_{33}$ component (see Supplementary Fig. S4, which shows a comparison to the vertical component of particle velocity), we can construct a comparison seismogram for the $\mathrm{e}_{11}$ component (see Methods). Figure 4 shows that the fit is excellent. Hence, for this event at this station, the signal is probably dominated by the horizontal strain component $\mathrm{e}_{11}$. As the vertical strain component is approximately related to the derivative of the Rayleigh wave eigenfunctions with depth beneath the virtual receiver, we infer that the eigenfunction is likely to be approximately constant with depth at the earthquake location.

A strike-slip virtual receiver such as earthquake 3 in Fig. 3 records the sum of the $e_{12}$ and $e_{21}$ components of the strain (Supplementary Methods, Equations (S30)-(S33)). In the Supplementary Discussion, Supplementary Fig. S2 shows that the virtual and real recordings of earthquake 1 using this strike-slip virtual receiver also compare well. Supplementary Fig. S5 shows the case when earthquake 2 is recorded on a virtual receiver constructed from earthquake 4 . In this geometry the Supplementary Methods shows that the virtual sensor records only the $e_{33}$ component, providing a fundamentally new measurement in seismology.

Previously, Hong and Menke ${ }^{25}$ estimated virtual seismograms by a different method. They added active source recordings together to generate pseudo-noise sequences and then applied the passive noise form of interferometry to estimate inter-source responses (that is, they sum over receivers, then cross-correlate). Unfortunately, accurate seismogram construction from passive noise requires much longer time series 
than are afforded by typical earthquake seismograms ${ }^{23}$, and consequently in Supplementary Fig. S5 we show that their method produces less accurate seismogram approximations. Our approach is different: we use the impulsive source form of interferometry by first cross-correlating responses and only then summing over receivers. This requires only the actual, recorded seismograms at each receiver.

Although we formulated theory only for acoustic and elastic wave propagation (see Supplementary Methods), this can be extended into forms appropriate for diffusive, attenuating, electro=magnetic or electro-kinetic energy propagation ${ }^{11-14}$. It is applied here to earthquake sources, but we could equally construct virtual sensors from fractures occurring in stressed solid material in a laboratory, or from impulsive pressure sources in liquid or gas, provided energy from such sources is recorded using an appropriately placed array of receivers.

The inter-earthquake seismogram is obtained by back-projecting data recorded from one earthquake through empirically recorded Green's functions from another, an explicit elastic expression of the acoustic time-reversal experiment of Derode and colleagues $^{29}$. However, the method also converts the data from particle displacement (or time derivatives thereof) at the real seismometers to strain due to seismic waves at the subsurface locations, the strain components matching those of the original source. Also, as this method essentially back-projects recordings to the virtual sensor location, it is equally possible to back-project other signals such as passive noise recordings to either or both of the pair of subsurface source locations. This offers the possibility of monitoring inter-earthquake Green's functions as a function of time either before or after the original earthquakes occurred, by using standard passive noise interferometry ${ }^{2-5}$.

In the exploration industry, seismic-frequency strain recordings have been shown to be particularly useful for wavefield analysis and subsurface imaging ${ }^{26,27}$. The direct, noninvasive sensitivity to strain provided by the virtual seismometers introduced here is the first such measurement within the interior of a solid. This holds promise for analysing stress or strain triggering of earthquakes by passing seismic waves, for example, as no other method has the potential to provide such deep or such widely distributed measurements of the strain field in the Earth's subsurface.

\section{Methods}


In the Supplementary Methods we present a general acoustic and elastic formulation for constructing virtual sensors using interferometry. We also develop theory for the particular case of an earthquake double-couple moment tensor source radiating Rayleigh and Love surface waves, as so far, seismic interferometry has derived useful information largely from the reconstruction of surface waves. We thus derive precisely which components of surface wave strain are recorded by virtual receivers constructed from canonical normal, thrust and strike-slip earthquakes, allowing verification of the method by comparison with directly recorded seismograms in these cases (Supplementary Table S1).

A potential problem in verifying virtual receiver seismograms (for example, in Fig. 4) is that no direct seismic frequency strain sensors exist in the Earth close to earthquakes for comparison. To make direct comparisons possible, in principle one could construct horizontal strain measurements by computing scaled differences between closely spaced seismometers ${ }^{27}$, but in the frequency range considered here (15-33 s period) across the southwestern US this is generally not possible because the seismometer distribution is spatially aliased. Instead we derive estimates of the scaled horizontal strain in a direction in line with the source-seismometer path by taking time derivatives of measured seismograms. This results in frequency domain multiplication by $i \omega=i c k$, where $\omega$ and $k$ are temporal and in-line spatial frequencies, respectively, and $c$ is the phase velocity. Thus we approximate a spatial derivative (multiplication by $i k$ ) assuming that the unknown phase velocity $c$ does not change rapidly within the frequency band considered (we also took account of the azimuth of propagation, which can change the sign of the horizontal strain estimates).

There is no equivalent operation for approximating vertical strains in the examples presented above. Vertical strain measurements from virtual receivers therefore constitute new information about Earth vibrations. If an earthquake is considered to be temporally impulsive with moment tensor $\mathbf{M}_{1}$ and is recorded by a virtual sensor constructed from another earthquake with moment tensor $\mathbf{M}_{2}$, the data consist of a sum of strain Green's functions between the locations of the two 
earthquakes, scaled by the product of the respective moment tensor components (Supplementary Methods, Equations (S15)-(S18)). However, earthquake sources are also generally non-impulsive. If $W_{i}(\omega)$ is the frequency domain representation of the source time function of earthquake $i$, the seismograms recorded at the virtual sensor are modulated by $W_{2} W_{1}^{*}$ (Supplementary Methods, Equations (S10) and (S11)). Hence, if for example the two source time functions were similar, $W_{2} \approx W_{1}$, the recorded data would consist of inter-earthquake strain Green's functions modulated by the autocorrelation of the source time function, shifted in time by $t_{2}-t_{1}$, where $t_{i}$ is the origin time of earthquake $i$. We remove that time shift in the results presented in this letter and in Supplementary Methods. As a consequence, compared with zerophase seismometer recordings, residual phase shifts in the virtual sensor records are caused by differences between the two source time functions $W_{1}$ and $W_{2}$.

Received 14 April 2009; accepted 28 July 2009; published online 30 August 2009

\section{References}

$1 \quad$ Bijwaard, H. \& Spakman, W., Non-linear global P-wave tomography by iterated

$2 \quad$ linearized inversion. Geophys. J. Int. 141 (1), 71-82 (2000).

2 Campillo, M. \& Paul, A., Long-Range Correlations in the Diffuse Seismic Coda. Science 299 (5606), 547 - 549 (2003).

3 Shapiro, N. \& Campillo, M., Emergence of broadband Rayleigh waves from correlations of the ambient seismic noise. Geophysical Research Letters 31, L07614 (2004).

4 Shapiro, N., Campillo, M., Stehly, L., \& Ritzwoller, M., High-Resolution Surface-Wave Tomography from Ambient Seismic Noise. Science 307, 1615-1617 (2005).

5 Gertstoft, P., Sabra, K.G., Roux, P., Kuperman, W.A., \& Fehler, M.C., Green's functions extraction and surface-wave tomography from microseisms in southern California. Geophysics 71 (no. 4), SI23-SI31 (2006). Claerbout, J.F., Synthesis of a layered medium from its acoustic transmission response. Geophysics 33 (no. 2), 264-269 (1968).

7 Wapenaar, K., Synthesis of an inhomogeneous medium from its acoustic transmission response. Geophysics 68 (no. 5) (2003).

$8 \quad$ Wapenaar, K. \& Fokkema, J., Reciprocity Theorems for Diffusion, Flow, and Waves. J. Appl. Mech. 71 (1), 145-150 (2004).

$9 \quad$ Wapenaar, K., Thorbecke, J., \& Draganov, D., Relations between reflection and transmission responses of three-dimensional inhomogeneous media. Geophys. J. Int.

156, 179-194 (2004). Wapenaar, K. \& Fokkema, J., Green's function representations for seismic interferometry. Geophysics 71, SI33-SI44 (2006).

11 Slob, E., Draganov, D., \& Wapenaar, K., Interferometric electromagnetic Green's functions representations using propagation invariants. Geophys. J. Int. 169, 60-80 (2007). 
Slob, E. \& Wapenaar, K., Electromagnetic Green's functions retrieval by crosscorrelation and cross-convolution in media with losses. Geophysical Research Letters 34, L05307-05301 - L05307-05305 (2007).

Snieder, R., Extracting the Green's function of attenuating heterogeneous media from uncorrelated waves. J. Acoust. Soc. Am. 121, 2637-2643 (2007).

Snieder, R., Wapenaar, K., \& Wegler, U., Unified Green's function retrieval by crosscorrelation; connection with energy principles. Phys. Rev. E 75, 036103 (2007). Bakulin, A. \& Calvert, R., Virtual Source: new method for imaging and 4D below complex overburden. 74th Annual International Meeting, SEG, Expanded Abstracts, 2477-2480 (2004).

Bakulin, A. \& Calvert, R., The virtual source method: Theory and case study. Geophysics 71, SI139-SI150 (2006).

Dong, S., He, R., \& Schuster, G., Interferometric prediction and least squares subtraction of surface waves. 76th Annual International Meeting, SEG, Expanded Abstracts, 2783-2786 (2006).

Snieder, R., Wapenaar, K., \& Larner, K., Spurious multiples in seismic interferometry of primaries. Geophysics 71, SI111-SI124 (2006).

Halliday, D.F., Curtis, A., van-Manen, D.-J., \& Robertsson, J., Interferometric surface wave isolation and removal. Geophysics 72 (no. 5), A69-A73 (2007).

Mehta, K., Bakulin, A., Sheiman, J., Calvert, R., \& Snieder, R., Improving the virtual source method by wavefield separation. Geophysics 72 (No. 4), pp. V79-V86 (2007). Halliday, D.F., Curtis, A., \& Kragh, E., Seismic surface waves in a suburban environment - active and passive interferometric methods. The Leading Edge 27 (no. 2), 210-218 (2008).

van Manen, D.-J., Robertsson, J.O.A., \& Curtis, A., Modeling of wave propagation in inhomogeneous media. Phys. Rev. Lett. 94, pp. 164301-164301 - 164301-164304 (2005).

van Manen, D.-J., Curtis, A., \& Robertsson, J.O.A., Interferometric modeling of wave propagation in inhomogeneous elastic media using time reversal and reciprocity. Geophysics 71 (No. 4), SI47-SI60 (2006).

van Manen, D.-J., Robertsson, J.O.A., \& Curtis, A., Exact wavefield simulation for finitevolume scattering problems. J. Acoustic. Soc. Am. Express Letters 122 (No. 4), pp. EL115-EL121 (2007).

Hong, T.-K. \& Menke, W., Tomographic investigation of the wear along the San Jacinto fault, southern California. Phys. Earths Planet. Inter. 155 (3-4), 236-248 (2006).

Curtis, A. \& Robertsson, J., Volumetric wavefield recording and near-receiver group velocity estimation for land seismics. Geophysics 67, 1602-1611 (2002).

Source times used for the seismometer recordings are those from the International Seismology Centre (ISC) catalogue; no Centroid Moment Tensor (CMT) source mechanism and timing was available.

Snieder, R., Extracting the Green's function from the correlation of coda waves: A derivation based on stationary phase. Phys. Rev. E 69, 046610 (2004).

Derode, A. et al., Recovering the Green's function from field-field correlations in an open scattering medium. J. Acoust. Soc. Am. 113 (6), 2973-2976 (2003).

Robertsson, J.O.A. \& Curtis, A., Wavefield separation using densely deployed, three component, single sensor groups in land surface seismic recordings. Geophysics $\mathbf{6 7}$ (no. 5) (2002). 


\section{FIGURES}
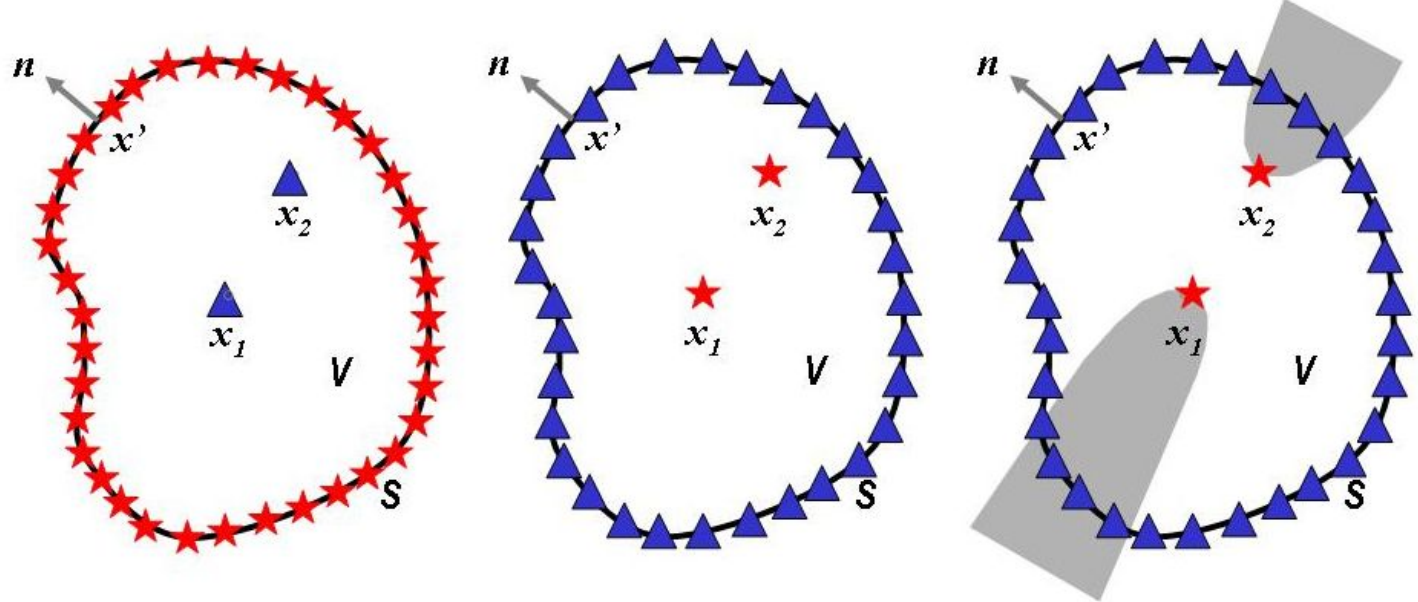

Figure 1: Schematic Overview of Seismic Interferometric Methods.

Left: To-date seismic interferometry estimates the Green's function between the locations of two receivers (triangles) at $x_{1}$ and $x_{2}$, by cross-correlating waves radiating from energy sources (stars), recorded on some boundary $S$ surrounding volume $V$. Centre: in the Supplementary Material we use reciprocity to approximatethe same Green's function given energy sources at $x_{1}$ and $x_{2}$ recorded at receivers on S. Right: Sneider ${ }^{28}$ showed that for either the left or centre case, the Green's function can be approximated using only receivers around the extension of the $x_{1}-x_{2}$ line (within the gray areas). 

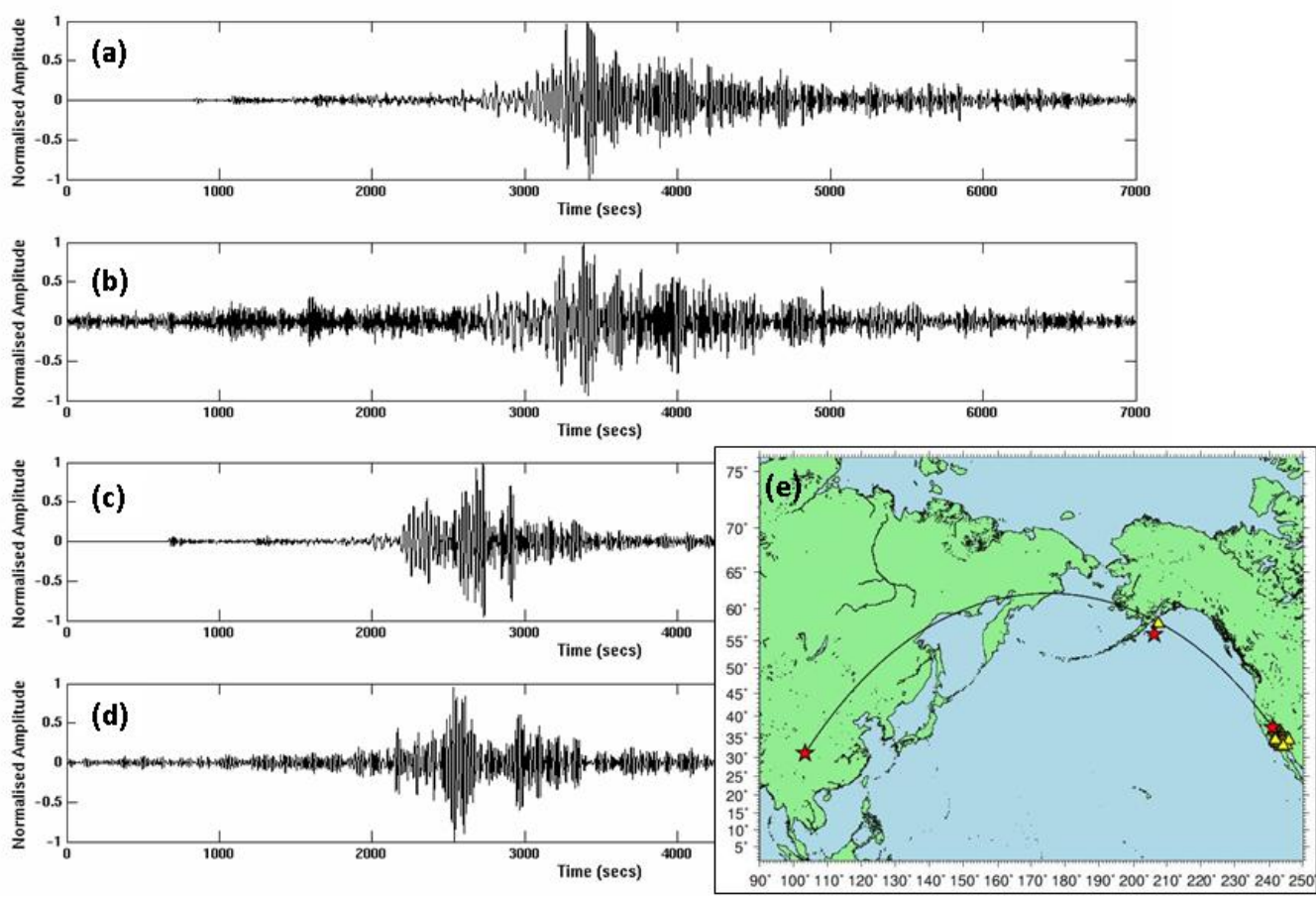

Figure 2. Comparison of Real and Virtual Recordings of the Sichuan Earthquake.

Comparison of virtual and real receiver recordings of 2008 Sichuan earthquake using the configuration in (e). (a) Real recording at MLAC in California; (b) virtual receiver recording at event within 40km of MLAC; (c) real recording at KDAK in Alaska; (d) virtual receiver recording at event within $260 \mathrm{~km}$ of KDAK. All vertical component. Inset: location map. Earthquakes (red stars); seismic stations (yellow triangles); great circle path (solid, black line). 


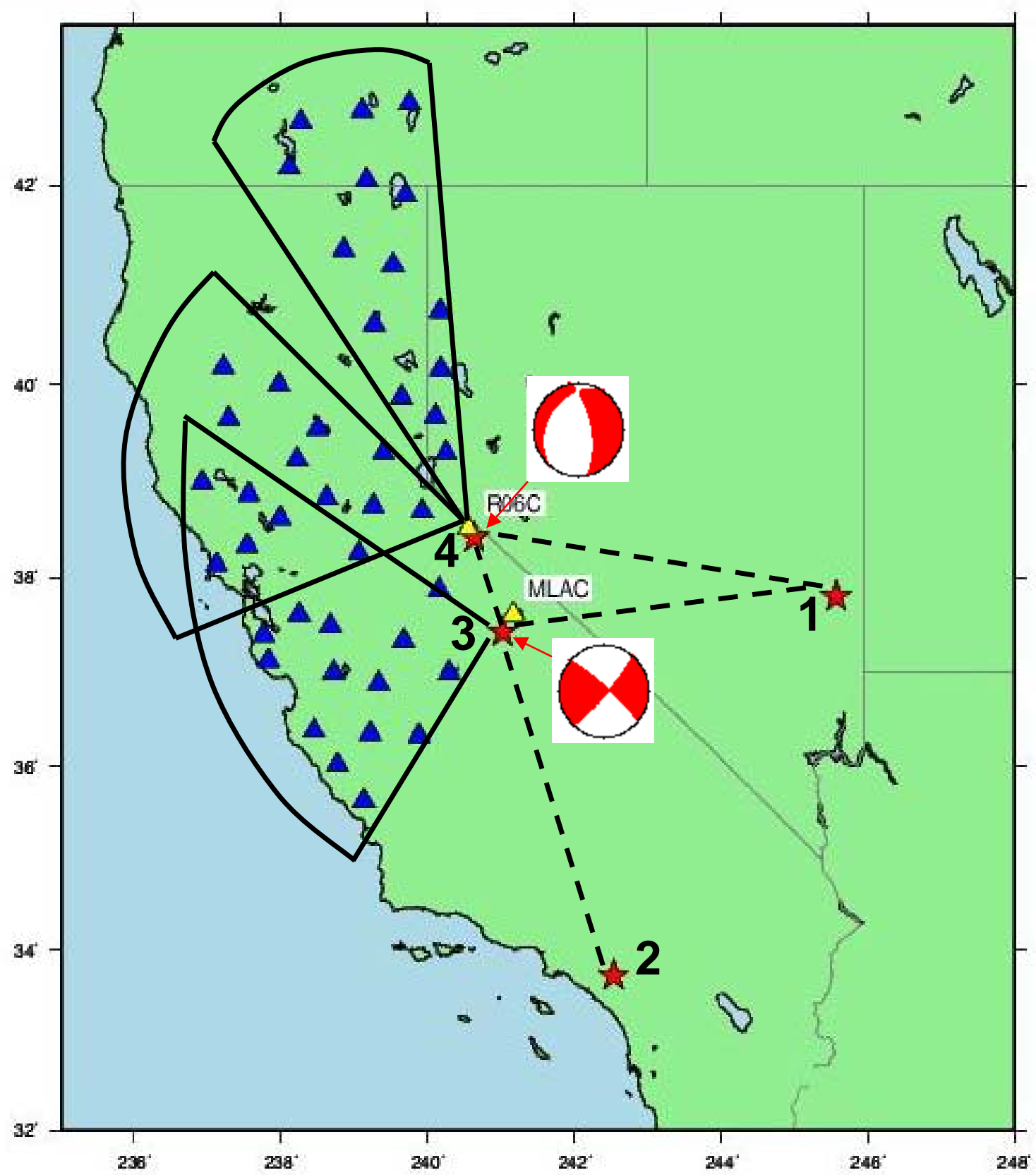

Figure 3. South-West USA Location Map.

Earthquakes (red stars) numbered 1 to 4 ; seismic stations used in interferometry (blue triangles); seismic stations for comparison (yellow triangles); focal mechanisms of virtual receivers are shown as standard lower-hemisphere projections near to their locations. Dashed lines indicate inter-Earthquake paths, solid lines connected by arcs indicate the region within which receivers were located for each Earthquake pair. 

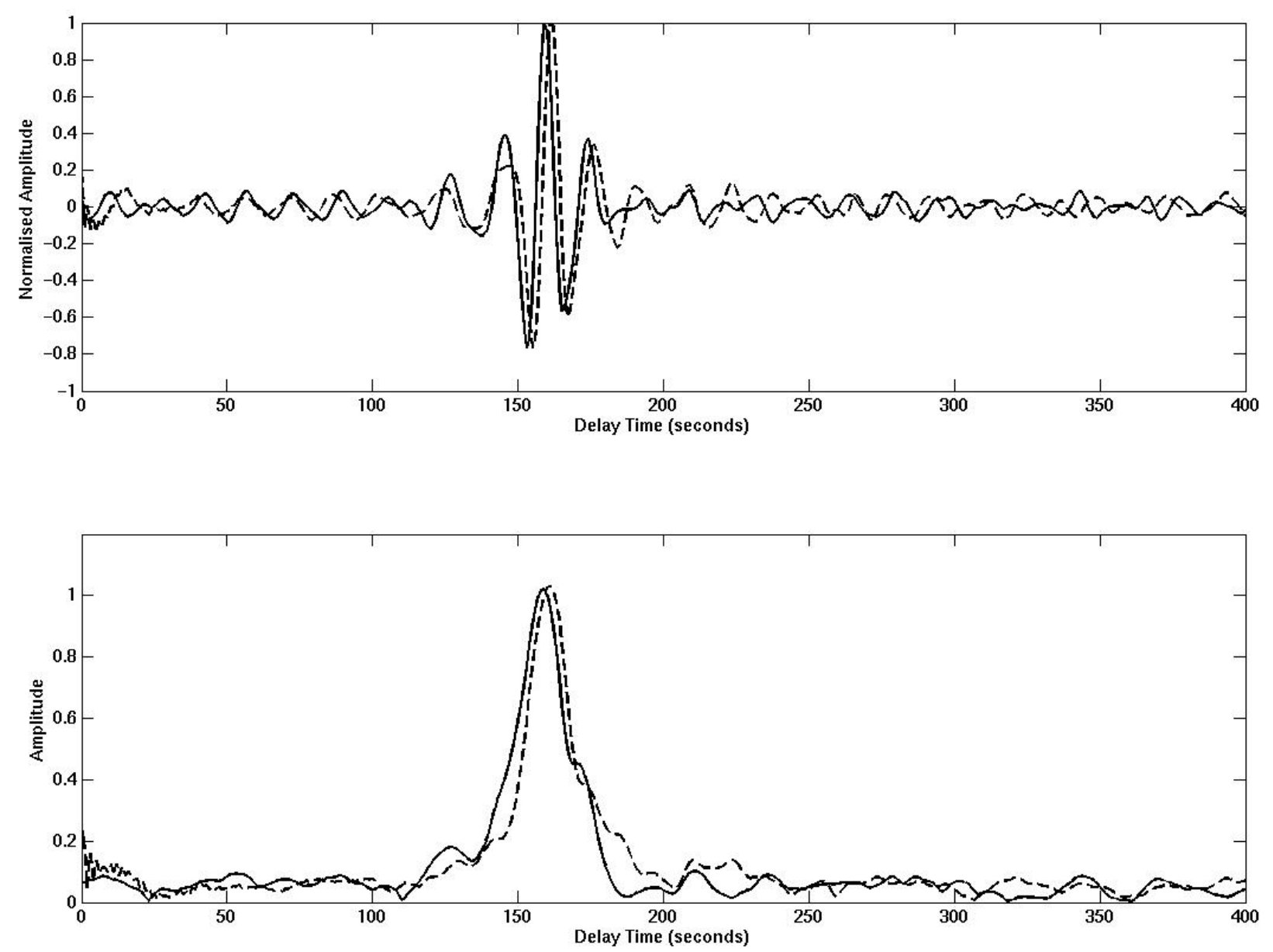

Figure 4. Comparison of Real and Virtual Recordings in California.

Comparison of seismograms (top) and envelope functions (bottom) of earthquake 1 recorded by the normal virtual receiver 4 (solid) with the directly-recorded, inverted, time derivative of the radial-component measurements from seismometer R06C (dashed). Virtual receiver records are constructed using 15 stations from the USArray and Berkeley seismic networks (Figure 3). 